Artículo científico

Volumen 30(3):613-630. Septiembre-diciembre, 2019 e-ISSN 2215-3608, doi:10.15517/am.v30i3.36027 http://www.revistas.ucr.ac.cr/index.php/agromeso

\title{
Evaluación ex-ante del impacto del genotipo de arroz L23 en la Altillanura plana colombiana ${ }^{1}$
}

\section{Ex-ante impact evaluation of the L23 rice genotype on the colombian Altillanura plains}

\author{
Diego Armando Marín-Salazar ${ }^{2}$, Robert Santiago Andrade-López ${ }^{3}$, Ricardo Antonio Labarta-Chávarri ${ }^{4}$, \\ Ángela Rocío Vásquez-Urriago ${ }^{5}$, Samuel Caicedo Guerrero ${ }^{6}$
}

1 Recepción: 31 de enero, 2019. Aceptación: 14 de mayo, 2019. Este trabajo formó parte del proyecto de investigación realizado bajo el convenio TV16-08 entre el Centro Internacional de Agricultura Tropical (CIAT) y la Corporación Colombiana de Investigación Agropecuaria (AGROSAVIA) en el desarrollo de estrategias tecnológicas y vinculación del conocimiento en los sistemas de producción de cultivos transitorios, en la Altillanura plana y piedemonte Llanero colombiano. Colombia.

2 Universidad del Valle y Centro Internacional de Agricultura Tropical (CIAT), Palmira, Colombia. d.marin@cgiar.org (https://orcid.org/00000001-5229-5355).

3 University of Minnesota y Centro Internacional de Agricultura Tropical (CIAT), Palmira, Colombia. r.s.andrade@cgiar.org (https://orcid. org/0000-0002-5764-3854).

4 Michigan State University y Centro International de Agricultura Tropical (CIAT), Palmira, Colombia. r.labarta@cgiar.org (https://orcid. org/0000-0003-3517-8768).

5 Universidad Complutense de Madrid y Corporación Colombiana de Investigación Agropecuaria (AGROSAVIA). Km 14 Vía Mosquera Bogotá, Cundinamarca, Colombia. arvasquez@agrosavia.co (https://orcid.org/0000-0002-5487-2950).

6 Colegio de Postgraduados de Chapingo y Corporación Colombiana de Investigación Agropecuaria (AGROSAVIA), Centro de Investigación La Libertad - Villavicencio, Meta, Colombia. scaicedo@agrosavia.co (https://orcid.org/0000-0002-5822-5881).

\section{Resumen}

Introducción. La Altillanura colombiana atraviesa por un proceso de transformación, marcado por la expansión de su frontera agrícola. En la región, se está implementando una agenda de investigación que busca desarrollar y validar tecnologías promisorias para el mejoramiento de la productividad y la sostenibilidad de los sistemas de producción agropecuarios. La evaluación económica juega un papel importante en priorizar actividades, asignar recursos y guiar la investigación agrícola hacia objetivos de desarrollo rural. Objetivo. El objetivo de este trabajo fue implementar una evaluación económica ex-ante para estimar los resultados e impactos potenciales de la nueva línea promisoria de arroz L23 desarrollada para la Altillanura colombiana. Materiales y métodos. El impacto se midió en el genotipo de arroz L23, desarrollado en colaboración por la Corporación Colombiana de Investigación Agropecuaria (AGROSAVIA) y el Centro Internacional de Agricultura Tropical (CIAT), para la Altillanura colombiana. La evaluación ex-ante efectuada combinó un análisis de presupuestos parciales con información de pruebas experimentales y un modelo de excedentes económicos. Resultados. Los dos enfoques metodológicos fueron consistentes y mostraron que con la adopción del genotipo L23, en al menos 29,7\%, se podría conseguir una rentabilidad del 17,1\% para los productores de arroz de la Altillanura, debido al aumento esperado del rendimiento en el cultivo. Sin embargo, un análisis de sensibilidad de los supuestos, alerta que un bajo nivel potencial de adopción junto a cambios no significativos en rendimiento convertiría la investigación y desarrollo del nuevo genotipo en no factible a nivel económico. Conclusión. Para que la investigación, desarrollo e introducción del genotipo de arroz L23 sea rentable, se requiere un proceso de 
diseminación que permita la reproducción de la semilla y facilite el acceso a los productores, para que así puedan alcanzar mayores niveles de adopción.

Palabras clave: costos de producción, evaluación de tecnologías, variedades, rentabilidad, toma de decisiones.

\begin{abstract}
Introduction. The Colombian Altillanura is going through a process of transformation, marked by the expansion of its agricultural border. In the region, it is being implemented a research agenda that seeks to develop and validate promising technologies for the improvement of productivity and sustainability of agricultural production systems. Economic evaluation plays an important role in prioritizing activities, allocating resources, and guiding agricultural research towards rural development objectives. Objective. The objective of this work was to implement an ex-ante economic evaluation to estimate the results and potential impacts of the new promising L23 rice line improved for the Colombian Altillanura. Materials and methods. The impact was measured on the L23 rice genotype, which was developed as a collaboration between the Colombian Corporation of Agricultural Research (AGROSAVIA) and the International Center for Tropical Agriculture (CIAT), for the Colombian Altillanura. The ex-ante evaluation combined a partial budgets analysis with information from experimental tests and economic surpluses model. Results. The two methodological approaches were consistent and showed that with the adoption of the L23 genotype at least $29.7 \%$, a probability of $17.1 \%$ could be achieved for rice producers in the Altillanura due to the expected increase in crop yield. However, a sensitivity analysis of the assumptions warns that a low potential level of adoption along with nonsignificant changes in yield would make the research and development of the new genotype economically unfeasible. Conclusion. For the research, development and, introduction of the L23 rice genotype to be profitable, it is required a diffusion process that allows the reproduction of the seed and facilitates the access to producers, so that they can reach higher levels of adoption.
\end{abstract}

Keywords: production costs, technology assessment, varieties, profitability, decision making.

\title{
Introducción
}

La investigación agrícola y el desarrollo tecnológico son actividades económicas que emplean recursos (físicos, humanos y operativos) escasos y valiosos, los cuales deben ser priorizados y optimizados. La evaluación económica ex-ante contribuye a priorizar, asignar recursos y guiar la investigación agrícola hacia objetivos de desarrollo rural (Castro, 1991).

En Colombia, la investigación agrícola se ha dirigido principalmente hacia regiones que permitan expandir potencialmente la frontera agrícola nacional. Se ha buscado establecer sistemas productivos que se ajusten a las condiciones climáticas, de suelo y otros factores de estas regiones. En las últimas décadas, la investigación agrícola ha puesto énfasis en la región de la Altillanura colombiana, que cuenta con una extensión de tierra cultivable que asciende a 3,5 millones de hectáreas, pero que es parte de un ecosistema que puede degradarse fácilmente. Dado este contexto, se hace prioritario avanzar la investigación sobre sistemas de producción agropecuarios modernos y sostenibles (Gómez, 2010).

Dado que la Altillanura está atravesando un proceso marcado por una fuerte expansión de su frontera agrícola (Abadía, 2011), se ha generado una alta demanda de tecnologías que deben ser adecuadas para una región que enfrenta diversas limitaciones para el acceso de sus recursos naturales (calidad de suelos, destrucción de bosques y pasturas nativas, escasez de agua, entre otros) (Gómez, 2010). Para la investigación agrícola, es importante 
conocer el impacto económico potencial que podrían generar nuevas tecnologías agrícolas en caso de ser adoptadas. Este análisis es aún más relevante dada la limitación de recursos que existe para llevar a cabo las actividades de investigación agrícola. En el caso de la Altillanura, los esfuerzos se han concentrado en desarrollar actividades de investigación que potencien la biodiversidad del trópico húmedo de la región (García, 2009); además de temas relacionados con el aumento de la productividad agropecuaria, manejo sostenible de los suelos, reducción de riesgos climáticos e introducción de variedades promisorias de cultivos adaptadas a las condiciones edáficas y climáticas de la región (Soler y Hernández-Rodríguez, 2018).

En respuesta a las necesidades de la región y las diversas implicaciones que tiene la expansión de la frontera agrícola, de una manera responsable y sostenible, el Ministerio de Agricultura y Desarrollo Rural (MADR), la Corporación Colombiana de Investigación Agropecuaria (antes CORPOICA y ahora denominada AGROSAVIA desde junio de 2018) y el Centro Internacional de Agricultura Tropical (CIAT), suscribieron un convenio en el año 2011 con el principal objetivo de desarrollar y validar tecnologías para el mejoramiento de la productividad y la sostenibilidad de los sistemas de producción agropecuarios en la Altillanura plana de la Orinoquía colombiana.

Algunas de las tecnologías desarrolladas están orientadas al cultivo del arroz, el cual está entre los más importantes del país. Según la encuesta nacional agropecuaria del año 2016, el arroz es el tercer cultivo de mayor extensión agrícola después del café y el maíz (DANE, 2016b), con un valor económico de su producción en paddy de 2,9 billones de pesos (950,5 millones de US\$). Este valor representa alrededor del $5 \%$ del Producto Interno Bruto Agropecuario Nacional (FAOSTAT, 2018). Por otra parte, el consumo per cápita de arroz blanco ha ido incrementando con un promedio alrededor de 39,6 kg anuales, entre 2009 y 2015. La mayor parte del consumo nacional es producido en Colombia, sin embargo, la cantidad importada ha estado creciendo. Durante el 2015 y 2016 la cantidad importada representó el $12 \%$ y $13 \%$, respectivamente de la cantidad total consumida (FEDEARROZ, 2018). Este incremento en importaciones en parte se atribuye a las condiciones del TLC firmado con Estados Unidos en el 2012. Inicialmente el arroz ingresado tenía un arancel del $80 \%$ durante los primeros seis años, el cual irá disminuyendo, con un plazo de desgravación de diecinueve años. Según Tirado y Barreto (2014) el sector arrocero colombiano tiene bajos niveles de productividad y altos costos de producción, y una desventaja tecnológica en comparación con Estados Unidos, lo cual podría hacer más rentable la importación de arroz cuando haya completa desgravación que producirlo en territorio nacional. Así pues, el desafío para las instituciones de investigación CIAT, AGROSAVIA y MADR es fortalecer el sector arrocero nacional, por medio del desarrollo tecnológico, para reducir la brecha tecnológica y de productividad con otros países de la región, y mejorar la competitividad del sector.

Bajo el convenio MADR-CORPOICA-CIAT se implementó una encuesta a nivel de fincas durante el periodo 2011-2012, con el objetivo de entender los sistemas productivos de la región de la Altillanura (Rodríguez-Borray et al., 2015); esta encuesta permitió una caracterización de la zona y la identificación de algunas de las limitantes que padecen los productores de la región. El trabajo abarcó un total de 120 encuestas, las cuales fueron recopiladas en los municipios de Puerto Gaitán (59\%) y Puerto López (41\%), se abarcó esta área que corresponde al 7,25 \% de la superficie cultivable de la Altillanura colombiana. En el caso específico del cultivo del arroz, solo catorce productores (el 11,6\% del total de la muestra) estaban cultivando este cereal al momento de la encuesta. A pesar de que el arroz es uno de los principales cultivos transitorios, el área total registrada alcanzó tan solo 6089 ha, lo cual representa 2,4 \% del área total que abarcó la encuesta. Del análisis de este grupo se pudieron determinar algunas características relevantes que pueden ser generalizables a la región; por ejemplo, una de las variedades más sembrada en la zona es la Línea 30 (relanzada como Corpoica Llanura 11 en el 2011) que viene siendo utilizada a partir de su creación en 1991. Otras de las variedades mencionadas en uso en la región y en la encuesta fueron la Fedearroz 174 y Fedearroz 757, las cuales no son las más adecuadas para un sistema de producción de secano, como el predominante en la zona. 
El desarrollo de variedades de arroz altamente productivas y adaptadas a las condiciones de la región fue una prioridad del convenio MADR-CORPOICA-CIAT; y se le encargó al programa de arroz del CIAT el proceso de mejoramiento genético. Inicialmente se creó una población con 55 líneas que proporcionaban una diversa y amplía base genética, que fue mejorada mediante el método de selección recurrente; la estrategia de mejoramiento de la población de germoplasma de arroz secano estuvo enfocada a las características de resistencia a la Pyricularia, la adaptabilidad a suelos ácidos y potencial de rendimiento. El método de selección recurrente constó de la selección de las mejores plantas que tenían los caracteres de interés, conformando nuevas poblaciones cada vez más pequeñas con líneas élites. Al final, mediante el método de selección pedigrí, los científicos del programa de arroz seleccionaron tres líneas segregantes promisorias, una de estas fue la L23 (Cubillos, 2017).

En el año 2016, la línea L23 se sometió a un proceso agronómico y de evaluación llevado a cabo por CORPOICA en sus estaciones experimentales y fincas de la región. En noviembre de 2017, CORPOICA obtuvo la autorización de registro de este genotipo, en el registro nacional de cultivares comerciales del Instituto Colombiano Agropecuario (ICA), con el objetivo de iniciar su liberación en la región de la Altillanura. Finalmente, a través de la resolución ICA No. 00024795 del año 2018, se registró la línea L23 como la nueva variedad Corpoica Porvenir 12.

La evaluación ex-ante se implementó usando modelos económicos que se parametrizaron en función de la opinión de expertos y una revisión bibliográfica intensiva. Este tipo de evaluación económica considera los elementos críticos de alternativas tecnológicas para retroalimentar el proceso de toma de decisiones (Moñux et al., 2005).

Varios autores sugieren utilizar el método de presupuestos parciales para identificar alternativas tecnológicas agrícolas promisorias con mayor rentabilidad en diferentes cultivos transitorios y perennes (Botero et al., 1990; Domínguez et al., 2016); este método centra su análisis en la variación de los costos de insumos, mano de obra y maquinaria, entre las diferentes alternativas tecnológicas evaluadas (CIMMYT, 1988).

Una vez identificadas las alternativas tecnológicas promisorias, el método de excedentes económicos es usado de manera frecuente para estimar los beneficios económicos de la inversión en investigación agrícola (Rivas y Herrera, 2003; Hareau et al., 2006), lo cual según Castro (1991), se logra mediante un análisis de costo-beneficio, basado en indicadores de rentabilidad para asignar recursos entre alternativas o proyectos, con el fin de maximizar impactos.

El objetivo de este trabajo fue implementar una evaluación económica ex ante para estimar los resultados e impactos potenciales de la nueva línea promisoria de arroz L23 desarrollada para la Altillanura colombiana.

\section{Materiales y métodos}

A partir de la colaboración entre la Corporación Colombiana de Investigación Agropecuaria (AGROSAVIA) y el Centro Internacional de Agricultura Tropical (CIAT), se realizó inicialmente un análisis de presupuestos parciales, seguido por un análisis de excedentes económicos, que incorpora las condiciones del mercado de arroz a nivel nacional. Dadas las características del mercado nacional, el método de excedentes económicos se implementó a partir del supuesto de funciones lineales de la oferta y la demanda en una economía cerrada (Alston et al., 1995) dadas las características del mercado nacional.

\section{Presupuesto parcial}

El presupuesto parcial es un método de análisis económico que ha sido utilizado por varias décadas, principalmente en ensayos experimentales agrícolas (Perrin et al., 1983; CIMMYT, 1988). Un enfoque ampliamente usado en estudios de evaluación económica ex ante de tecnologías en el cultivo de arroz (Annou et al., 2000; Gianessi et al., 2002; Bond et al., 2003), que considera pruebas experimentales de una nueva tecnología 
e información representativa de la tecnología convencional. Esta metodología evalúa alternativas tecnológicas con promedios de rendimiento diferentes, las cuales presentan costos distintos y, generalmente muestran una relación positiva entre los costos y los beneficios. Los costos que difieren entre una opción tecnológica y otra se denominan costos que varían, y se diferencian de los otros costos que permanecen constantes independientemente de la tecnología usada, los cuales no son relevantes para la identificación de alternativas más rentables.

En el presupuesto parcial primero se deben calcular los costos relevantes, para lo cual se deben multiplicar los costos unitarios de los insumos relevantes para cada alternativa tecnológica por la intensidad de uso; después se determina el precio de la producción que es igual al que podría vender el agricultor su producto antes de cosecharlo. Se considera el precio de mercado del producto restando el costo unitario de cosecha y comercialización; el cual se multiplica por el rendimiento del cultivo, para obtener el beneficio bruto de la producción, que al ser restado de los costos que varían y los fijos se convierten en beneficio neto. Al final, se realiza un análisis de dominancia entre las alternativas o tratamientos, en donde se define a un tratamiento como dominado cuando tiene beneficios netos menores o iguales a los de un tratamiento con costos que varían más bajos; entonces, deben identificarse todos los tratamientos que son dominados y descartarlos para el análisis posterior. Luego, para el cálculo de la tasa de retorno marginal (TRM), se trabaja con los tratamientos no dominados, y se obtiene al dividir el incremento de los beneficios sobre el aumento de los costos.

La tasa mínima de retorno (TMR), permite conocer el costo de uso del capital utilizado por un agricultor para financiar el tratamiento (alternativa tecnológica). Finalmente, la regla de decisión sugiere que la inversión en una nueva tecnología es económicamente viable si el retorno económico (TRM) es mayor al que recibiría el agricultor por depositar el mismo capital en el mercado financiero o en otra tecnología.

\section{Análisis de excedentes económicos}

En un trabajo pionero en evaluación de tecnologías agrícolas, Griliches (1957) sugirió que el perfil de una tecnología sigue un comportamiento de tipo logístico, con una fase inicial que corresponde a los primeros años de introducción de la tecnología, caracterizada por niveles bajos de adopción; posteriormente, cuando los agricultores conocen de la tecnología, las tasas de adopción crecen a un ritmo más rápido hasta llegar a un nivel máximo, allí se mantienen por un periodo hasta experimentar un proceso de desadopción u obsolescencia. En esta evaluación, la adopción de tecnología agrícola está definida como un proceso dinámico con diferentes fases a través del tiempo.

El método de análisis de excedentes económicos intenta medir el aumento de la productividad o reducción de costos asociados a la adopción de una nueva tecnología; supone que las curvas de oferta $\left(Q_{s}\right)$ y demanda $\left(Q_{D}\right)$ son lineales en un equilibrio parcial de mercado (Figura 1). El proceso de difusión y diseminación de la tecnología provocaría un desplazamiento vertical paralelo de la curva de oferta, que genera un nuevo punto de equilibrio (P1, Q1) (Figura 1). Una vez que se determina este desplazamiento de la curva de oferta se puede aproximar el cambio en el bienestar de los agentes que intervienen en el mercado a causa de la tecnología, a través del excedente del productor y del consumidor. El desplazamiento de la curva de oferta deja evidente una reducción en el costo unitario de producción, que conduce a un aumento de las cantidades producidas y consumidas $(\Delta \mathrm{Q}=\mathrm{Q} 1-\mathrm{Q} 0)$ y una caída de los precios $(\triangle \mathrm{P}=\mathrm{P} 1-\mathrm{P} 0)$.

Los excedentes del consumidor están representados por el área $P_{0} P_{I} b a$, mientras el excedente del productor es igual al área $P_{l} d c b$; este último será positivo si la reducción de los costos unitarios por adoptar la nueva tecnología son mayores que la caída del precio. El cambio en el excedente total es $I_{0} I_{1} b a$ y es equivalente al área $P_{0} d c b a$, debido a la ley de equivalencia de paralelogramos. Para esta investigación se utilizó la formulación planteada por Alston et al. (1995). 


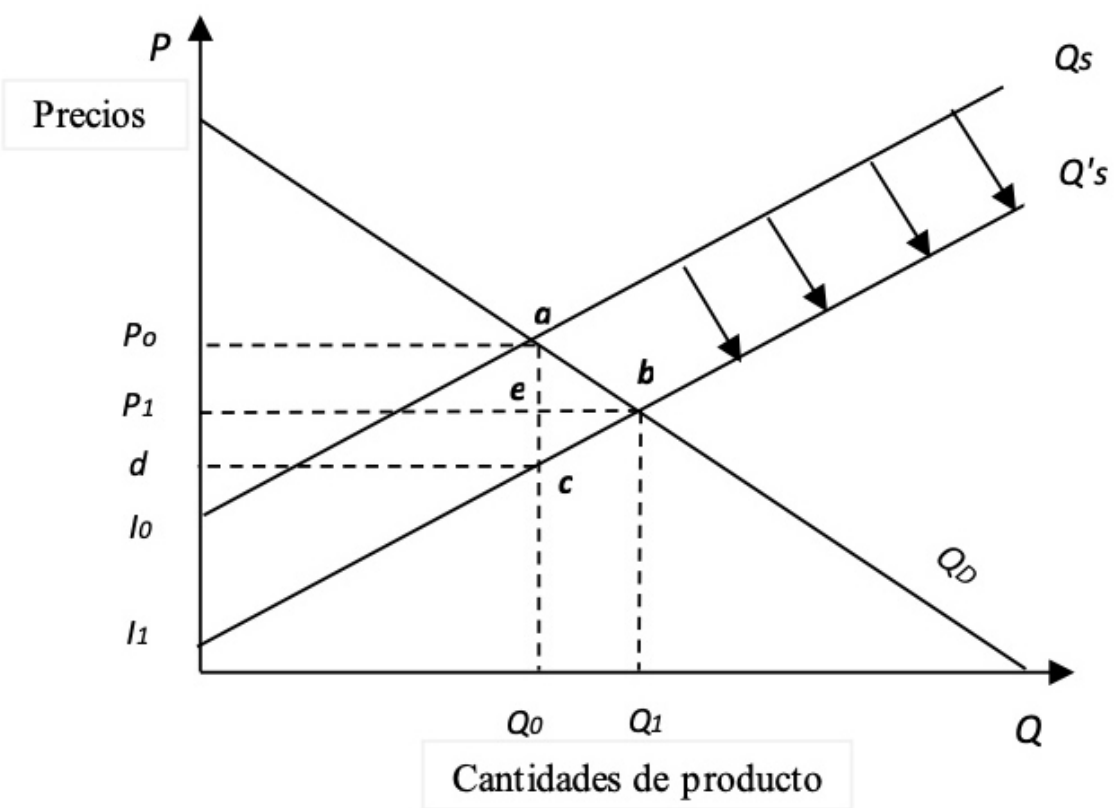

Figura 1. Desplazamiento vertical de la curva de oferta por introducción de nueva tecnología. Fuente: Alston et al. (1995).

Figure 1. Vertical shift of the supply curve due to the introduction of new technology. Source: Alston et al. (1995).

\section{Información utilizada y definición de parámetros de elasticidades, precios y cantidades iniciales}

Los parámetros económicos utilizados en los modelos con función de oferta y demanda lineal permiten caracterizar el mercado del arroz a través del tiempo, y muestran las condiciones del precio y cantidad inicial de equilibrio antes de la introducción de la nueva variedad. Mediante los parámetros técnicos de adopción, cambio de productividad, variación en costos y elasticidades de oferta y demanda, se caracteriza el mercado del arroz después de la liberación de la variedad y, por medio de la simulación, los nuevos precios y cantidades de equilibrio se estiman como consecuencia de la adopción tecnológica.

El horizonte temporal para realizar el análisis y establecer los beneficios económicos potenciales fue establecido en veintiséis años, distribuidos de la siguiente manera: se considera un periodo de inversión inicial entre el año 2011 y 2016 (periodo de seis años), en donde se contabilizan los costos de investigación y desarrollo (I y D) por parte de AGROSAVIA y CIAT, para el desarrollo de la nueva línea promisoria de arroz L23 (aclarando que previo al convenio ya se tenían poblaciones de germoplasma registradas de arroz de secano) (Guimarães et al., 1995).

El análisis de los beneficios y su distribución entre los diferentes actores de este mercado se hace a partir del año 2017, fecha donde se registra L23 en el ICA, hasta el año 2037, suponiendo un ciclo de uso de veinte años, según los supuestos de sustitución tecnológica más comunes (Alston et al., 1995); se estima que, luego de estos veinte años se inicia un proceso de desadopción y la línea mejorada es remplazada por una nueva tecnología, período que no se incluye en este análisis. En el Cuadro 1 se resumen los principales parámetros, sus valores y principales fuentes de información.

Después de calcular los excedentes económicos en el horizonte temporal definido en veintiséis años, el análisis financiero del flujo de fondos a partir de indicadores de rentabilidad, tal como la tasa interna de retorno (IRR) y el Valor Presente Neto (VPN), permiten conocer la viabilidad económica del desarrollo de la nueva línea de arroz 
Cuadro 1. Parámetros de elasticidades, precios y cantidades producidas de arroz. Región de la Altillanura plana de la Orinoquía, Colombia. 2016-2017.

Table 1. Parameters of elasticities, prices, and quantities of rice produced. Altillanura region of the Orinoquía, Colombia. $2016-2017$.

\begin{tabular}{|c|c|c|c|}
\hline Parámetros & Valor & Unidad & Fuente \\
\hline Elasticidad precio de la oferta & 0,94 & $\mathrm{dQ} \mathrm{dP}^{-1}$ & Ramírez et al. (2004) \\
\hline Elasticidad precio de la demanda & $-0,45$ & $\mathrm{dQ} \mathrm{dP}^{-1}$ & Ramírez et al. (2004) \\
\hline $\begin{array}{l}\text { Producción inicial de la región (sin } \\
\text { cambio tecnológico) }\end{array}$ & 24144 & $\mathrm{t}$ & Cálculos propios a partir de DANE (2016a) \\
\hline $\begin{array}{l}\text { Costo de producción (sin cambio } \\
\text { tecnológico) }\end{array}$ & 317,2 & $\mathrm{US} \$ \mathrm{t}^{-1} *$ & FEDEARROZ (2016) \\
\hline $\begin{array}{l}\text { Precio de equilibrio (sin cambio } \\
\text { tecnológico) }\end{array}$ & 361,7 & US\$ $\mathrm{t}^{-1 *}$ & FEDEARROZ (2016) \\
\hline $\begin{array}{l}\text { Rendimiento Arroz (sin cambio } \\
\text { tecnológico) }\end{array}$ & 3,1 & $\mathrm{t} \mathrm{ha}^{-1}$ & Tapiero et al. (2011) \\
\hline $\begin{array}{l}\text { Rendimiento Arroz (con cambio } \\
\text { tecnológico) }\end{array}$ & 3,5 & $\mathrm{t} \mathrm{ha}^{-1}$ & $\begin{array}{l}\text { Expertos CORPOICA, Centro de Investigación La } \\
\text { Libertad (comunicación personal, 2017) }\end{array}$ \\
\hline $\begin{array}{l}\text { Costo de producción (con cambio } \\
\text { tecnológico) }\end{array}$ & 301,4 & $\mathrm{US} \$ \mathrm{t}^{-1 *}$ & $\begin{array}{c}\text { Expertos CORPOICA, Centro de Investigación La } \\
\text { Libertad (comunicación personal, 2017). Reducción } 5 \\
\% \text { de costos por resistencia a Pyricularia) }\end{array}$ \\
\hline
\end{tabular}

*Los precios fueron constantes al año 2016 / *Prices were constant prices to the year 2016.

L23. Para el cálculo de los indicadores se utiliza una tasa social de descuento uniforme del $12 \%$, manejada en Colombia para todos los proyectos de inversión (Piraquive et al., 2018). Existen criterios de decisión para rechazar o aprobar la inversión: si el VPN arroja un valor positivo o si la IRR es mayor a la tasa mínima de rentabilidad del $12 \%$, la inversión se considera viable.

El cálculo de la IRR tiene problemas cuando en los flujos intermedios de caja se produce más de un cambio de signo, es decir, el proyecto tiene flujos de caja negativos diferentes a los del año cero (inversión) (Bora, 2015), por lo cual, se propone una tasa interna de retorno modificada (MIRR), que iguala el valor presente de los flujos de efectivo negativos al valor futuro de los flujos de efectivo positivo. La MIRR supone que los flujos de efectivo se reinvierten a la tasa requerida de rendimiento y no como la IRR tradicional, que supone se reinvierten a la misma IRR del proyecto (Solé, 2011). Para esta investigación se consideró una tasa de reinversión del 3,5 \%, según la tasa de interés real media ponderada del World Bank (World Bank, 2016).

\section{Perfil de adopción de la línea promisoria de $\operatorname{arroz} L 23$}

Es importante anticipar el nivel potencial de la adopción de la nueva línea promisoria de arroz; para ello, se han definido algunos parámetros que permiten construir dos escenarios del perfil de adopción. Para obtener estos parámetros se diseñó una encuesta, la cual se aplicó a los investigadores expertos de AGROSAVIA, sobre las expectativas de adopción en diferentes momentos del tiempo y así, poder simular este proceso.

El primer perfil asume una distribución logística sigmoidal con una adopción inicial del 2,7 \% en el año 1 de la liberación de la tecnología y que, según los expertos, alcanzaría un techo máximo del 29,7 \% a los diez años de haber sido liberada (Cuadro 2). El segundo perfil asume una distribución similar, pero con una adopción inicial del 2,6 \% y una máxima del 64,7 \% alcanzada a los trece años después de la liberación de este genotipo (Figura 2). Este perfil de adopción es propuesto bajo el supuesto de que la L23 reemplazará la variedad Corpoica Llanura 11 (Línea 
Cuadro 2. Perfil de adopción de la línea promisoria de arroz L23. Región de la Altillanura plana de la Orinoquía, Colombia. $2016-2017$.

Table 2. Adoption profile of the promising rice line L23. Altillanura region of the Orinoquía, Colombia. 2016-2017.

\begin{tabular}{lcc}
\hline & & Tecnología \\
\cline { 2 - 3 } Concepto & L23 (Perfil 1) & L23 (Perfil 2) \\
\hline Año inicio adopción & 2017 & 2017 \\
Nivel inicial de adopción & $2,7 \%$ & $2,6 \%$ \\
Año donde alcanza la máxima adopción & 2027 & 2030 \\
Máximo nivel adopción & $29,7 \%$ & $64,7 \%$ \\
Año de inicio de la obsolescencia & No obsolescencia & No obsolescencia \\
Año de desadopción total & No desadopción & No desadopción \\
\hline
\end{tabular}

Fuente: Expertos de CORPOICA, Centro de Investigación La Libertad / Source: Experts from CORPOICA, La Libertad Research Center.

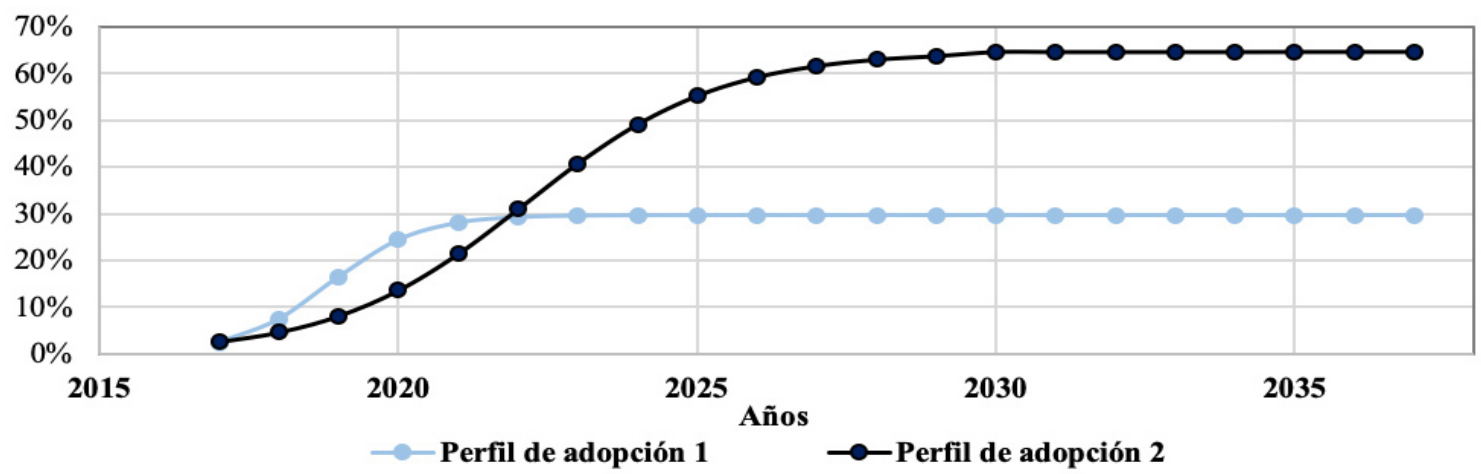

Figura 2. Curvas de adopción de la línea promisoria de arroz L23. Región de la Altillanura plana de la Orinoquía, Colombia. 20162017.

Figure 2. Adoption curves of the promising rice line L23. Altillanura region of the Orinoquía, Colombia. 2016-2017.

30), semilla (no registrada) que tuvo un proceso de difusión en los años 90's; su registro formal fue en el 2011 y su lanzamiento al año siguiente. En 2011 alcanzó el nivel de adopción propuesto como el nivel máximo (64 \%), según encuestas de 120 fincas levantadas en el convenio de MADR, CORPOICA y CIAT suscrito en el 2011. Se asume que el nuevo genotipo L23 podría alcanzar este mismo nivel de adopción.

\section{Escenarios planteados}

Para el análisis se definieron dos escenarios considerando distintos cambios en el rendimiento y los costos generados por la nueva línea promisoria, que podrían reflejar su mejor comportamiento agronómico cuando sea usada por los agricultores. El primer escenario está basado en la opinión de expertos de AGROSAVIA, mientras el segundo escenario es un promedio de los cinco ensayos experimentales realizados en terrenos de la zona de Altillanura (Cuadro 3). 
Cuadro 3. Escenarios potenciales por el uso de la línea promisoria de arroz L23. Región de la Altillanura plana de la Orinoquía, Colombia. 2016-2017.

Table 3. Potential scenarios for the use of promising rice line L23. Altillanura region of the Orinoquía, Colombia. $2016-2017$.

\begin{tabular}{|c|c|c|c|c|}
\hline Escenario & $\begin{array}{l}\text { Variedad } \\
\text { evaluada }\end{array}$ & $\begin{array}{c}\text { Cambio en rendimiento } \\
\Delta\end{array}$ & Cambio en costos $\Delta$ & Adopción de tecnología \\
\hline Escenario 1 & L23 & $12,9 \%$ & $-5,0 \%$ & Perfil 1 \\
\hline Escenario 1' & L23 & $12,9 \%$ & $-5,0 \%$ & Perfil 2 \\
\hline \multicolumn{2}{|c|}{ Fuente } & $\begin{array}{l}\text { Expertos CORPOICA, Centro } \\
\text { de Investigación La Libertad } \\
\text { (comunicación personal, 2017) }\end{array}$ & $\begin{array}{l}\text { Expertos CORPOICA, Centro } \\
\text { de Investigación La Libertad } \\
\text { (comunicación personal, 2017) }\end{array}$ & \\
\hline Escenario 2 & $\mathrm{~L} 23$ & $1,1 \%$ & $-0,5 \%$ & Perfil 1 \\
\hline Escenario 2' & $\mathrm{L} 23$ & $1,1 \%$ & $-0,5 \%$ & Perfil 2 \\
\hline \multicolumn{2}{|c|}{ Fuente } & $\begin{array}{l}\text { Pruebas semicormerciales } \\
\text { CORPOICA, año 2016. Terreno: } \\
\text { todos los ensayos experimentales }\end{array}$ & $\begin{array}{l}\text { Pruebas semicormerciales } \\
\text { CORPOICA, año 2016. Terreno: } \\
\text { todos los ensayos experimentales }\end{array}$ & \\
\hline
\end{tabular}

\section{Costos de investigación y desarrollo de las líneas promisorias}

Para estimar los beneficios que generarían la investigación y el desarrollo de la nueva línea promisoria de arroz, es necesario conocer el flujo de costos asumidos por AGROSAVIA y el CIAT para su desarrollo. La Figura 3 muestra la información suministrada por las dos instituciones, ajustada a precios constantes del 2017.

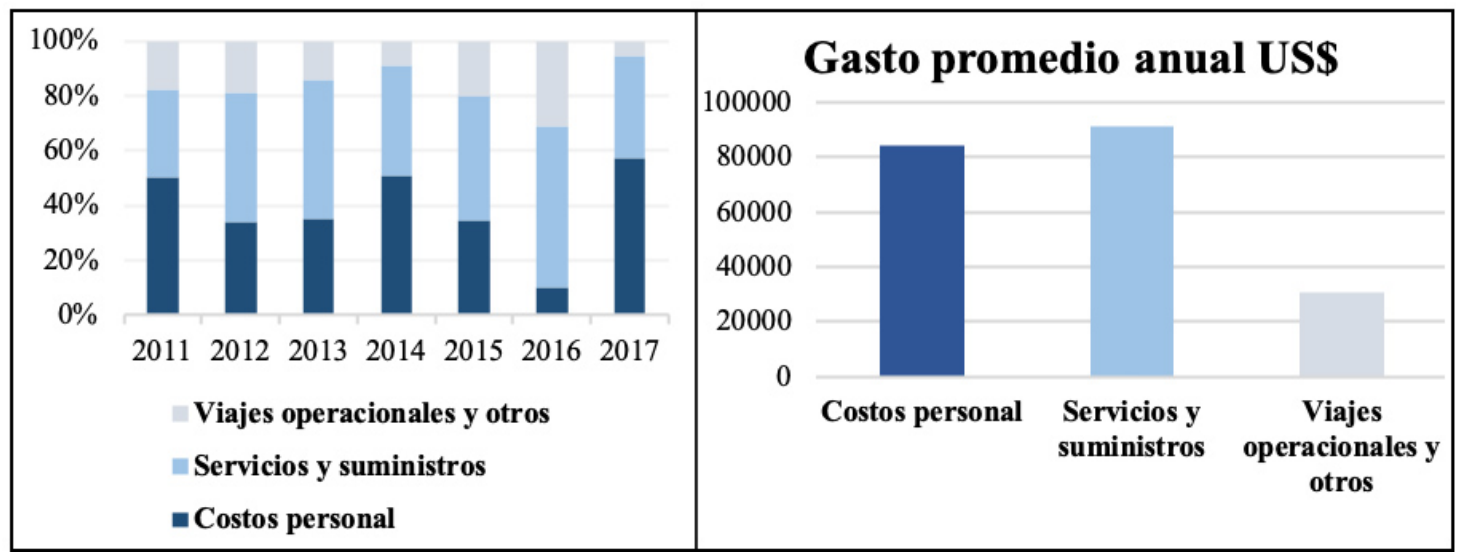

Los precios fueron constantes al año 2016 / Prices were constant throughout the year 2016.

Figura 3. Gastos en investigación de CORPOICA y CIAT por actividades, para el desarrollo de la línea promisoria de arroz L23. Región de la Altillanura plana de la Orinoquía, Colombia. 2016-2017.

Figure 3. Research expenditures of CORPOICA and CIAT by activities, for the development of the promising rice line L23. Altillanura region of the Orinoquía, Colombia. 2016-2017.

Entre el 2011 y el 2014, el CIAT ejecutó la mayor parte del gasto de su presupuesto, principalmente en gastos de personal, servicios y suministros. En este periodo se concentraron los esfuerzos en el desarrollo genético de las 
nuevas líneas promisorias de arroz. En los años 2015 y 2016 CIAT redujo los gastos en un 48,2 \% pasando de 243 900 dólares en el año 2014 a 124800 dólares en 2015 y a 82800 dólares en 2016. Durante estos últimos años, solo se establecieron ensayos experimentales para medir la eficiencia agronómica de esta línea de arroz y pruebas semicomerciales, actividades a cargo de CORPOICA. Al final, el costo total registrado hasta el 2017 del desarrollo de la nueva línea de arroz estuvo alrededor de 1050000 dólares.

\section{Resultados}

\section{Análisis de presupuesto parcial}

En el presupuesto parcial se compararon dos alternativas tecnológicas: la variedad tradicional de arroz Corpoica Llanura 11 y la línea promisoria de arroz L23; dentro del análisis, se definió la variedad tradicional como línea de base y se preparó una estructura de costos e indicadores financieros basados en la información obtenida de los ensayos experimentales, pruebas de evaluación agronómica y pruebas semi-comerciales realizadas por CORPOICA, en cinco localidades, en el año 2016 (Cuadro 4).

Cuadro 4. Estructura de costos e indicadores financieros de las pruebas semi-comerciales de la variedad de arroz CORPOICA Llanura 11 (caso de línea base). Región de la Altillanura plana de la Orinoquía, Colombia. 2016-2017.

Table 4. Cost structure and financial indicators of the semi-commercial tests of the rice variety CORPOICA Llanura 11 (base line case). Altillanura region of the Orinoquía, Colombia. 2016-2017.

\begin{tabular}{|c|c|c|c|c|}
\hline Concepto & Unidad & $\begin{array}{l}\text { Centro de Investigación } \\
\text { La Libertad }\end{array}$ & $\begin{array}{l}\text { Centro de Investigación } \\
\text { Carimagua }\end{array}$ & $\begin{array}{c}\text { Otros ensayos } \\
\text { (promedio de tres) }\end{array}$ \\
\hline Preparación terreno & US\$ ha-1 & 57,4 & 54,1 & 43,2 \\
\hline Siembra & US\$ ha-1 & 151,2 & 151,2 & 151,2 \\
\hline Enmiendas y fertilizantes & US\$ ha ${ }^{-1}$ & 201,4 & 384,2 & 310,4 \\
\hline Control de malezas & US\$ ha ${ }^{-1}$ & 146,4 & 75,6 & 65,2 \\
\hline Control de plagas & US\$ ha ${ }^{-1}$ & 19,3 & 98,7 & 83,8 \\
\hline Control de enfermedades & US\$ ha ${ }^{-1}$ & - & - & - \\
\hline Cosecha & US\$ ha ${ }^{-1}$ & 114,3 & 209,1 & 152,8 \\
\hline $\begin{array}{l}\text { Costos fijos (Fomento arrocero } 0,5 \% \text { del } \\
\text { valor de la producción) }\end{array}$ & US\$ ha-1 & 87,3 & 56,2 & 55 \\
\hline Total costos & US\$ ha-1 & 777,2 & 1029,1 & 861,6 \\
\hline Rendimiento (kg ha-1) & kilos ha-1 & 3286 & 4312 & 3611 \\
\hline $\begin{array}{l}\text { Precio al productor arroz paddy verde } \\
\text { (Molinos de Villavicencio) }\end{array}$ & US\$ kg-1 & 0,33 & 0,33 & 0,33 \\
\hline Ingresos (ha) & US\$ ha ${ }^{-1}$ & 1068,4 & 1402 & 1174,08 \\
\hline Margen neto (ha) & US\$ ha ${ }^{-1}$ & 291,3 & 372,9 & 312,47 \\
\hline Rentabilidad & $\%$ & $27,30 \%$ & $26,6 \%$ & $26,6 \%$ \\
\hline
\end{tabular}

Fuente: Datos no publicados del año 2016 de CORPOICA. Pruebas Semi-comerciales a diferentes genotipos de arroz para la Altillanura colombiana / Source: Unpublished data of the year 2016 of CORPOICA. Semi-commercial tests for different genotypes of rice for the Colombian Altillanura. 
Para la nueva variedad de arroz L23 también se realizaron ensayos experimentales, pruebas de evaluación agronómica y semi-comerciales en las mismas localidades. Se identificaron los costos relevantes (costos que varían) que muestran la diferencia entre una variedad y otra.

En el análisis de presupuestos parciales para el genotipo L23 en las cinco localidades evaluadas, se encontró que los costos de manejo agronómico no variaron con respecto a la variedad Corpoica Llanura 11, excepto los relacionados con la cuota de fomento arrocero y los costos de cosecha. Estos últimos comprenden las actividades de servicio de corte y transporte de la producción que está influenciado por el rendimiento y la ubicación de los predios donde se hizo la evaluación.

Los resultados de rendimiento y costos difirieron entre localidades. Los indicadores de productividad y rentabilidad sugirieron que las pruebas realizadas en el Centro de Investigación (C.I.) La Libertad y C.I. Carimagua evidenciaron ventajas por el uso de la L23, con un incremento de los rendimientos del 13,8 \% y $35 \%$ y un margen neto del $49 \%$ y $119 \%$, respectivamente. Asimismo, la rentabilidad creció en 8 puntos porcentuales en C.I. la Libertad y 16,5 puntos en el C.I. Carimagua.

Los ensayos agronómicos realizados en la sede de CORPOICA Taluma, finca Andremoni y Miti-Miti, no evidenciaron una mejora en el rendimiento del nuevo genotipo de arroz sobre la variedad Corpoica Llanura 11 (Figura 4). La disminución en los costos, si bien existe, no es significativa y está asociada principalmente a los rubros de cosecha y cuota de fomento arrocero, y costos relacionados a la producción.

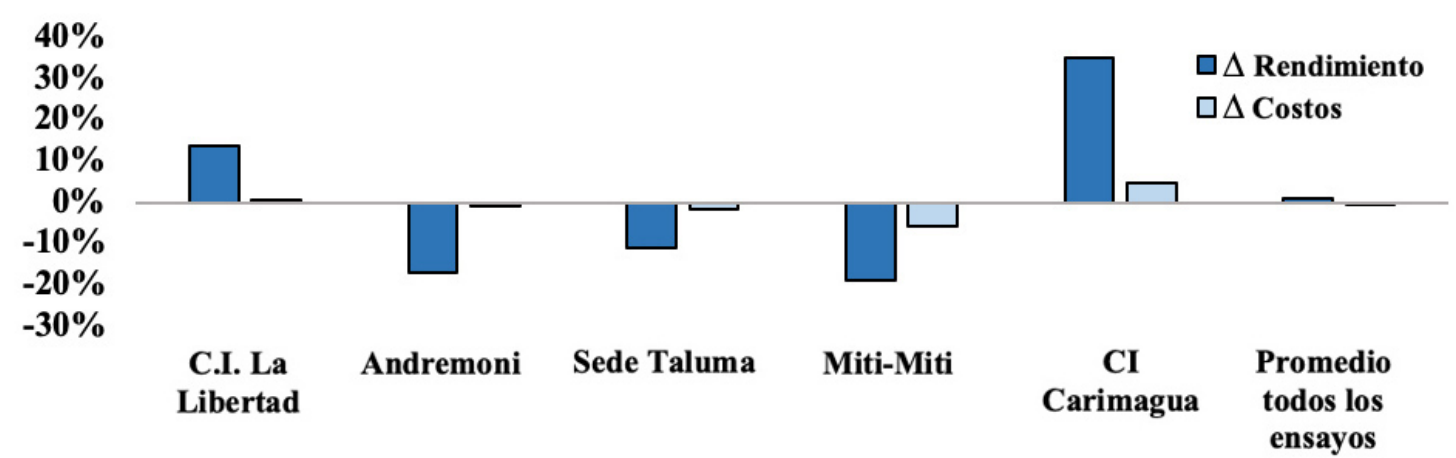

Fuente: Datos no publicados del año 2016 de CORPOICA. Pruebas semi-comerciales de diferentes genotipos de arroz para la Altillanura colombiana / Source: Unpublished data of the year 2016 of CORPOICA. Semi-commercial tests for different genotypes of rice for the Colombian Altillanura.

Figura 4. Cambio en rendimiento y costos por el uso de la línea promisoria de arroz L23, en cinco ensayos experimentales. Región de la Altillanura plana de la Orinoquía, Colombia. 2016-2017.

Figure 4. Change in yield and costs for the use of the promising L23 rice line in five experimental trials. Altillanura region of the Orinoquía, Colombia. 2016-2017.

Al realizar un análisis de dominancia, los ensayos experimentales realizados en otras localidades fueron alternativas dominadas, donde se corroboró mediante indicadores económicos que L23 sería una tecnología no viable (Cuadro 5). Por otro lado, si bien el ensayo en el C.I. Carimagua del genotipo L23 aumentó en 119 \% el margen neto, la tasa de retorno marginal (TRM) indicó que el uso de este genotipo generaría mayor rentabilidad 
Cuadro 5. Costos que varían, cambio en el margen neto y rentabilidad por el uso de la variedad L23 respecto a la variedad Corpoica Llanura 11, por localización de los ensayos experimentales. Región de la Altillanura plana de la Orinoquía, Colombia. 2016-2017.

Table 5. Change in costs, net margin, and profitability for the use of L23 variety regarding the variety Corpoica Llanura 11 by location of experimental trials. Altillanura region of the Orinoquía, Colombia. 2016-2017.

\begin{tabular}{|c|c|c|c|c|c|c|c|}
\hline \multirow[b]{2}{*}{ Concepto } & \multirow[t]{2}{*}{ Unidad } & \multicolumn{2}{|c|}{ C.I. La Libertad } & \multicolumn{2}{|c|}{ C.I. Carimagua } & \multicolumn{2}{|c|}{ Otros ensayos** } \\
\hline & & $\begin{array}{c}\Delta \\
\text { (cambio) }\end{array}$ & $\begin{array}{c}\Delta \\
(\text { cambio \%) }\end{array}$ & $\begin{array}{c}\Delta \\
\Delta \\
(\text { cambio \$) }\end{array}$ & $\begin{array}{c}\Delta \\
(\text { cambio \%) }\end{array}$ & $\begin{array}{c}\Delta \\
(\text { cambio } \$) \\
\end{array}$ & $\begin{array}{c}\Delta \\
(\text { cambio \%) } \\
\end{array}$ \\
\hline Cosecha & US\$ ha-1 & 4,46 & 4 & 44,51 & 21,3 & $-24,24$ & $-15,9$ \\
\hline $\begin{array}{l}\text { Costos fijos (Fomento } \\
\text { arrocero } 0,5 \% \text { del valor de la } \\
\text { producción) }\end{array}$ & US\$ ha-1 & 0,737 & 1 & 2,45 & 4,4 & $-0,191$ & $-1,7$ \\
\hline Total costos & US\$ ha-1 & 5,20 & 1 & 46,96 & 4,6 & $-25,19$ & $-2,9$ \\
\hline Rendimiento & kilos ha-1 & 454 & 14 & 1509 & 35,0 & -585 & $-16,2$ \\
\hline Ingresos & US\$ ha ${ }^{-1}$ & 147,61 & 14 & 490,63 & 35 & $-190,09$ & $-16,2$ \\
\hline Margen neto & US\$ ha ${ }^{-1}$ & 142,41 & 49 & 443,66 & 119 & $-164,89$ & $-52,8$ \\
\hline Rentabilidad $\Delta$ & Puntos \% & 8 & 16,5 & $-11,62$ & & & \\
\hline TMR & $\%$ & & 38 & & & Tratamiento & dominados \\
\hline
\end{tabular}

Comparativo frente a la variedad tradicional Corpoica Llanura 11 / Comparative with traditional variety Corpoica Llanura 11.

** Ensayos realizados en: Sede de CORPOICA de Taluma, Andremoni y Miti-Miti / ** Tests carried out in: CORPOICA Headquarters of Taluma, Andremoni and Miti-Miti.

Fuente: Datos no publicados del año 2016 de CORPOICA. Pruebas semi-comerciales de diferentes genotipos de arroz para la Altillanura colombiana / Source: Unpublished data of the year 2016 of CORPOICA. Semi-commercial tests for different genotypes of rice for the Colombian Altillanura.

cuando se siembre en el C.I. La Libertad. En ambos casos se satisface la regla de decisión de la TRM mayor a la tasa mínima de retorno (TAMIR) del $98 \%$ para Colombia, que corresponde a la tasa de interés en el mercado financiero informal que está alrededor del $58 \%$ EA (proveedores) (FINAGRO, 2014), sumado a la tasa de retorno mínimo exigido en otro cultivo alternativo al arroz (40 \% según Perrin et al. (1983).

\section{Análisis de excedentes económicos}

La estimación de los excedentes económicos que se generarían por la introducción de una nueva línea promisoria de arroz en la Altillanura colombiana, se realizó a partir de los parámetros especificados en la sección correspondiente de materiales y métodos. Estos excedentes son derivados de la reducción de precios de oferta, mayor producción disponible en el mercado y menores costos de producción.

Los excedentes calculados por la introducción del nuevo genotipo de arroz L23, varían dependiendo del escenario que se modele, y muestran sensibilidad cuando se consideran diferentes magnitudes en los cambios en rendimiento y costos y el perfil de adopción utilizado. Se definió el escenario (1) incorporando las expectativas de los expertos del comportamiento agronómico de la L23. Según su opinión, el rendimiento aumentaría en 12,9\% y los costos de producción se reducirían hasta en un $5 \%$; reducción que está relacionada a los costos de control de plagas y enfermedades, debido a la resistencia que este genotipo tiene hacia la Pyricularia.

Los resultados promedio en rendimiento y en la estructura de costos que arrojaron las pruebas semi-comerciales y agronómicas realizadas en cinco localidades, fueron considerados como un escenario (2) para el cálculo de los excedentes económicos, bajo el supuesto que los productores de arroz de la Altillanura obtendrán resultados 
similares por el uso de esta nueva variedad. Este escenario 2 supone un aumento en rendimientos de $1,1 \%$ y una reducción en costos de $0,5 \%$.

La Figura 5 muestra que, dada la simulación del comportamiento del mercado que hace este modelo basado en los parámetros iniciales y en el nivel de adopción que se asume (perfil de adopción 1 o 2, Figura 2), el uso de la nueva variedad generaría excedentes positivos a los productores de arroz, debido a que el efecto de la reducción de costos de producción y el aumento de rendimiento sería mayor que la disminución esperada de los precios de mercado. Asimismo, los resultados mostraron que existirían excedentes positivos a los consumidores, obtenidos a partir de la posibilidad que tendrían éstos de comprar mayores cantidades de arroz a un menor precio, gracias a la introducción de la nueva variedad; sin embargo, los resultados fueron variables entre escenarios, siendo el escenario 1 el que generaría los mayores excedentes al productor.

Suponiendo un perfil de adopción logístico sigmoidal sugerido por los expertos de AGROSAVIA (perfil de adopción 1), los resultados mostraron que, en cuanto a la distribución de los excedentes entre los diferentes actores de la economía, serían los consumidores los más beneficiados. En cualquiera de los dos escenarios, los beneficios de los consumidores serían más del doble de los que generarían los productores. Adicionando los excedentes obtenidos por los productores y consumidores, se tienen los excedentes totales que alcanzaría la sociedad en su conjunto. La Figura 5 muestra que para el escenario 1 los excedentes totales serían mayores en $952 \%$ frente al escenario 2. Los indicadores financieros (Cuadro 6) respaldan la ventaja del escenario 1, muestran la potencial viabilidad económica de la investigación y desarrollo de la L23, pues la tasa interna de retorno simple (IRR) y la modificada (MIRR) que considera una tasa de financiamiento del 3,5\%, según la tasa de interés real pública media ponderada del World Bank (World Bank, 2016), se calcularon en más del $12 \%$ correspondiente a la tasa social de descuento. Adicionalmente, el NPV fue positivo y la relación costo/beneficio se hizo mayor a 1. Por el contrario, bajo el escenario 2, la L23 no sería viable económicamente, dado que los indicadores financieros no respaldarían la inversión en investigación y desarrollo de este genotipo.

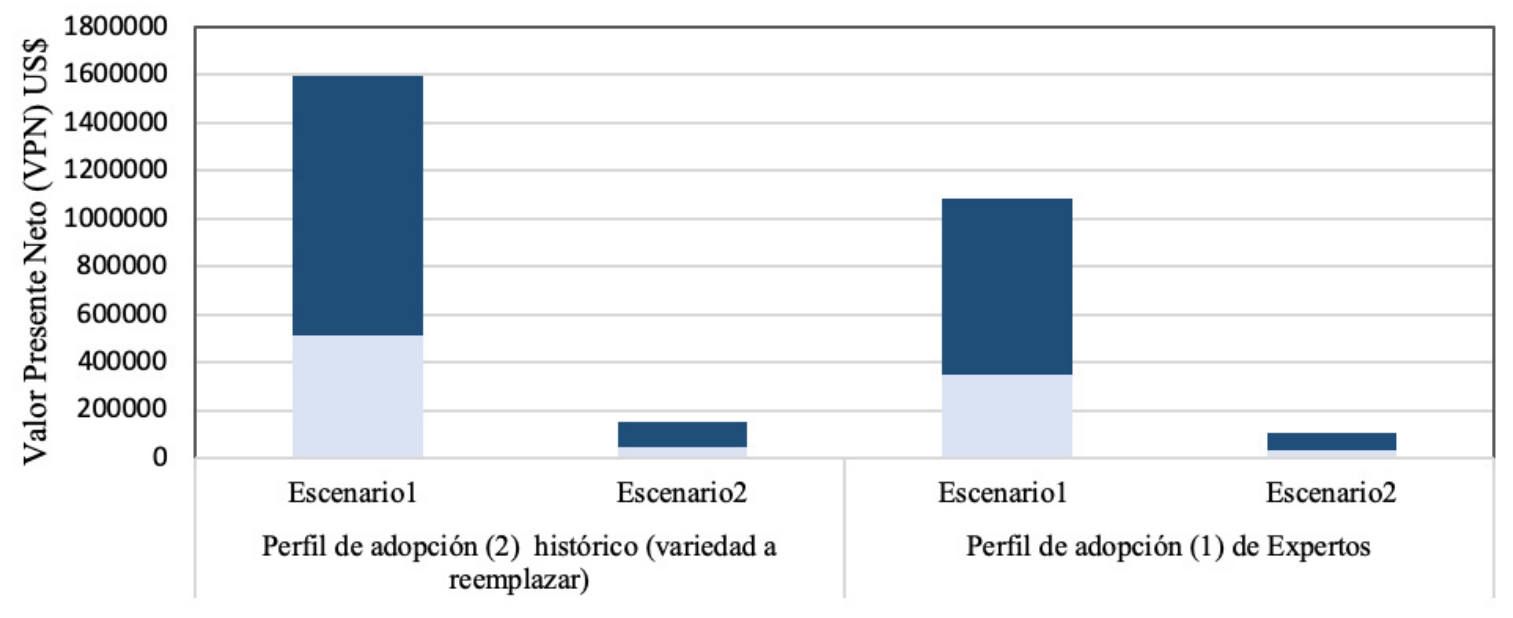

$\Delta$ Excedente Productor US\$ $\square \Delta$ Excedente Consumidor US\$

Figura 5. Excedentes económicos generados por la línea promisoria de arroz L23 bajo dos perfiles de adopción. Región de la Altillanura plana de la Orinoquía, Colombia. 2016-2017.

Figure 5. Economic surpluses generated by the promising line of rice L23 under two adoption profiles. Altillanura region of the Orinoquía, Colombia. 2016-2017. 
Cuadro 6. Indicadores económicos, rentabilidad de la línea promisoria de arroz L23. Región de la Altillanura plana de la Orinoquía, Colombia. 2016-2017.

Table 6. Economic indicators and profitability of the promising rice line L23. Altillanura plains region of the Orinoquía, Colombia. 2016-2017.

\begin{tabular}{|c|c|c|c|c|c|c|}
\hline $\begin{array}{c}\text { Perfil de } \\
\text { adopción Línea } \\
\text { L23 }\end{array}$ & Escenarios & $\begin{array}{c}\text { Cambio en } \\
\text { el excedente } \\
\text { económico total } \\
\text { (US\$) }\end{array}$ & IRR & $\begin{array}{c}\text { MIRR (tasa } \\
\text { de descuento } \\
12 \% \text { - Tasa de } \\
\text { reinversión 3,5 \%) }\end{array}$ & $\begin{array}{c}\text { NPV (tasa de } \\
\text { descuento 12 \%) } \\
\text { (US\$) }\end{array}$ & $(\mathrm{B} / \mathrm{C})$ \\
\hline \multirow{2}{*}{$\begin{array}{l}\text { Perfil de adopción } \\
\text { (1) Expectativas } \\
\text { de Expertos } \\
\text { AGROSAVIA }\end{array}$} & $\begin{array}{c}\text { Escenario } \\
1 \text { (Expertos } \\
\text { AGROSAVIA) }\end{array}$ & 1080935 & $17,2 \%$ & $13,2 \%$ & 419497 & 1,63 \\
\hline & $\begin{array}{c}\text { Escenario } \\
2 \text { (Ensayos } \\
\text { Experimentales }\end{array}$ & 102769 & $2,9 \%$ & $3,3 \%$ & -558668 & 0,16 \\
\hline $\begin{array}{l}\text { Perfil de adopción } \\
\text { (2) histórico } \\
\text { de variedad } \\
\text { tradiccional }\end{array}$ & $\begin{array}{c}\text { Escenario } \\
1 \text { (Expertos } \\
\text { AGROSAVIA) }\end{array}$ & 1595967 & $20,1 \%$ & $15,0 \%$ & 934529 & 2,41 \\
\hline Llanura 11 & $\begin{array}{c}\text { Escenario } \\
2 \text { (Ensayos } \\
\text { Experimentales }\end{array}$ & 151011 & $1,0 \%$ & $4,9 \%$ & -510426 & 0,23 \\
\hline
\end{tabular}

$\mathrm{IIR}=$ Tasa interna de retorno, $\mathrm{MIRR}=$ Tasa interna de retorno modificada, $\mathrm{NPV}=$ Valor presente neto, $\mathrm{B} / \mathrm{C}=\mathrm{Relación}$ costo-beneficio.$/$ $\mathrm{IIR}=$ Internal Rate of Return, MIRR= Internal Rate of Modified Return, NPV= Net Present Value, B/C= Cost-benefit ratio.

Suponiendo un perfil de adopción logístico donde la L23 reemplazará totalmente a la variedad tradicional Corpoica Llanura 11, alcanzando un máximo de adopción de 64,7 \% (perfil de adopción 2), como se esperaba, la magnitud de los excedentes sería mucho mayor a los obtenidos bajo el nivel de adopción (conservador) sugerido por los expertos, ya que la adopción se asume más alta, lo que generaría un desplazamiento de la curva de oferta más fuerte. El escenario 1 seguiría siendo el más viable, la inversión en investigación y desarrollo de la L23 tendría asociada una rentabilidad de $20 \%$, y unos excedentes totales para toda la economía de casi 1,6 millones de dólares. Sin embargo, nótese que bajo los supuestos del escenario 2, la inversión no tendría respaldo de los indicadores financieros, que sugeriría la no viabilidad económica del desarrollo del nuevo genotipo (Cuadro 6).

\section{Discusión}

Los resultados obtenidos a partir de la metodología de presupuestos parciales demostraron que es posible llegar a importantes consideraciones económicas, sujetas a la identificación de tratamientos de mayor rentabilidad, sin necesidad de contar con información completa de variables económicas, que como lo mencionan Ávalos-Cerdas y Villalobos-Monge (2018) implica un trabajo dificultoso de planeamiento, recolección y análisis que supera las posibilidades de los productores que cuentan con recursos escasos.

En este estudio se hallaron los costos que varían relacionados a costos de insumos, mano de obra y maquinaria, entre diferentes ensayos experimentales de la línea mejorada de arroz evaluada. Esta información económica es relevante porque como lo mencionan Perrin et al. (1983), permite al productor priorizar y tomar la decisión de manera precisa sobre la adopción de alternativas tecnológicas. El comportamiento de la reducción de costos de producción de la nueva línea de arroz L23, permite ver que si bien la productividad aumenta, los costos de producción también 
aumentan, como en el caso de los ensayos experimentales realizados en C.I. La Libertad y C.I. Carimagua que evidenciaron ventajas por el uso de la L23, con un incremento de los rendimientos del $13,8 \%$ y $35 \%$, pero con un aumento en costos del $2 \%$ y 4,6\%, respectivamente; esta situación es consistente con lo que expone Reyes-Hernández (2002), una relación directa entre los costos y beneficios de los tratamientos.

Aunque los presupuestos parciales es un método que implica un análisis económico con información incompleta o no sistematizada, es necesario generar herramientas para que los productores puedan obtener información necesaria para realizar análisis económicos de mayor robustez. En este estudio se consideraron tratamientos con base en costos en experimentos en pequeñas áreas. Para futuras investigaciones los costos deberían considerar la perspectiva de los agricultores para conducir la evaluación económica, ya que usualmente los costos en experimentos son subestimados (Reyes-Hernández, 2002); sin embargo, el método es defendible en ausencia de datos a nivel de finca para una tecnología que aún no se ha introducido comercialmente (Bond et al., 2003).

El método de excedentes económicos permitió simular el incremento de rendimiento óptimo para alcanzar un punto de equilibrio (NPV=0) donde la introducción de la variedad promisoria L23 fuera rentable. Los resultados mostraron que bajo un perfil de adopción similar al histórico de la variedad tradicional Corpoica Llanura 11, con un máximo de adopción de 64,7 \%, la nueva línea sería rentable cuando el incremento del rendimiento es igual o superior al 7,1\%. Para el caso del perfil de adopción esperado por los expertos, con una distribución logística sigmoidal con un techo máximo del 29,7 \%, el aumento de los rendimientos debería ser igual o mayor al 10,5\%. Los análisis realizados anteriormente dieron muestra que este modelo de excedentes económicos es altamente sensible a distintos niveles de adopción de la tecnología desarrollada (Espinosa y Wiggins, 2003). En próximas investigaciones se podría utilizar tanto métodos deterministas como estocásticos para realizar un análisis de sensibilidad que considere la incertidumbre respecto a la posible adopción, para estimar rentabilidad potencial de una nueva tecnología (Bond et al., 2003).

\section{Conclusiones}

El nuevo genotipo de arroz L23 mostró en algunas pruebas experimentales una mejor productividad respecto a la variedad tradicional de arroz predominante en la Altillanura colombiana. Con el modelo de presupuestos parciales se estimó que de las cinco zonas donde se llevaron a cabo ensayos experimentales, solamente en dos sería rentable el cultivo de arroz con la variedad L23. En el C.I. La Libertada y C.I. Carimagua, se esperaría un incremento en rendimiento de $13,8 \%$ y $35 \%$ respectivamente, frente a la variedad tradicional Corpoica Llanura 11.

El incremento en los rendimientos derivados del uso de esta nueva variedad podría generar un desplazamiento de la curva de oferta y, con ello, importantes excedentes económicos para la región de la Altillanura. El resultado del cálculo del excedente económico mostró que la adopción del nuevo genotipo resultaría rentable económicamente y por tanto, su lanzamiento sería factible bajo algunos escenarios. Bajo el escenario planteado a partir de los ensayos experimentales que supone un aumento en rendimientos de $1,1 \%$ y una reducción en costos de $0,5 \%$, la introducción del nuevo genotipo no sería viable económicamente, sin importar el perfil de adopción que se asuma. Teniendo en cuenta un escenario basado en la opinión de expertos científicos de AGROSAVIA, que consideran un incremento en rendimiento del $12,9 \%$ y una reducción en costos del 4,56 \%, los resultados mostraron que sí habría viabilidad económica en la investigación y desarrollo de la L23.

En general, se podría señalar que para que la investigación, desarrollo e introducción del nuevo genotipo L23 sea rentable para la Altillanura, se requiere un proceso de diseminación que permita la reproducción de la semilla que facilite el acceso a los productores y pueda alcanzar mayores niveles de adopción. La viabilidad de la adopción de esta nueva tecnología está sujeta a factores exógenos al productor, como la infraestructura para la comercialización de la nueva variedad de arroz, servicios de extensión para generar conocimiento sobre la tecnología, disponibilidad 
de maquinaria y mano de obra. Nuevas políticas e intervenciones para incentivar la adopción y el uso continuado de esta tecnología de arroz, deberían considerar estos factores.

\section{Agradecimientos}

Los autores agradecen la participación de investigadores del Programa de Cultivos Transitorios de AGROSAVIA, principalmente a José Eurípides Baquero Peñuela, Jaime Bernal y Oscar Gallo por la información de las pruebas semicomerciales y agronómicas de la línea de arroz L23, y por proporcionar opiniones sobre las expectativas agronómicas y de mercado de este genotipo promisorio. También se extienden los agradecimientos a los investigadores del Programa de Arroz del CIAT: Fernando Correa, Cécile Grenier y Yolima Ospina, y a investigadores de la unidad de Evaluación de Impacto del CIAT.

\section{Literatura citada}

Abadía, J.G. 2011. Cambios en la cobertura del paisaje y fuerzas conductoras en los Llanos Orientales Colombianos (Puerto López, Meta), 1988-2007. Tesis Bach., Pontificia Universidad Javeriana, COL.

Alston, J., G. Norton, and P. Pardey. 1995. Science under scarcity: principles and practice for agricultural research evaluation and priority setting. Cornell University Press, London, GBR.

Annou, M., E. Wailes, and G. Cramer. 2000. Economic analysis of adopting Liberty Link rice (RCS-2000, Rice Situation and Outlook, 55-61). Department of Agriculture Economic Research Service, WA, USA.

Ávalos-Cerdas, J.M, y A. Villalobos-Monge. 2018. Análisis económico: un estudio de caso en Jatropha curcas L. mediante la metodología de presupuestos parciales. Agron. Mesoam. 29:95-104. doi:10.15517/ma.v29i1.27901

Bond, C.A., C.A. Carter, and Y.H. Farzin. 2003. Medium grains, high stakes: Economics of genetically modified rice in California. AgBioForum 6(4):146-154.

Bora, B. 2015. Comparison between net present value and internal rate of return. IJRFM 5(12):61-71.

Botero, R., J.V. Cadavid, L. Rivas, A. Monsalve, y I.R. Sanint. 1990. Análisis económico ex-ante en sistemas de producción asociados: cultivo arroz-pradera. CIAT, Cali, COL.

Castro, H.M. 1991. Métodos y modelos para priorizar la investigación agropecuaria. IICA, Turrialba, CRI.

CIMMYT. 1988. La formulación de recomendaciones a partir de datos agronómicos: Un manual metodológico de evaluación económica. CIMMYT, México D.F., MEX.

Cubillos, E. 2017. Listo genotipo de Arroz "L23" para ser comercializado en los Llanos orientales. Agronet MinAgricultura, COL. http://www.agronet.gov.co/Noticias/Paginas/Listo-genotipo-de-Arroz-L23-para-ser-comercializado-en-los-Llanosorientales---17-de-noviembre-de-2017.aspx (consultado 15 jun. 2018).

DANE (Departamento Administrativo Nacional de Estadística). 2016a. $4^{\circ}$ Censo nacional arrocero 2016, resultados. DANE, COL. https://www.dane.gov.co/files/investigaciones/agropecuario/censo-nacional-arrocero/presentacion-4to-censonacional-arrocero-2016.pdf (consultado 10 oct. 2017).

DANE (Departamento Administrativo Nacional de Estadística). 2016b. Encuesta Nacional Agropecuaria (ENA). https:// www.dane.gov.co/index.php/estadisticas-por-tema/agropecuario/encuesta-nacional-agropecuaria-ena/encuesta-nacionalagropecuaria-por-departamentos (consultado 8 may. 2019). 
Domínguez, J., J. González, y L. Pagliettini. 2016. Análisis económico de la producción de arroz orgánico en Entre Ríos y Corrientes, Argentina. Comparación con respecto a la producción convencional. Agron. Ambiente Rev. Fac. Agron. 36(1):53-71.

Espinosa, J.A., y S. Wiggins. 2003. Beneficios económicos potenciales de tecnología bovina de doble propósito en el trópico mexicano. Téc. Pecu. Méx. 41:19-36.

FAOSTAT. 2018. Crops. FAO, Rome, ITA. https://bit.ly/2q6A316. (accessed May 9, 2019).

FEDEARROZ (Federación Nacional de Arroceros). 2016. Área, producción y rendimiento. FEDEARROZ, COL. http://www. fedearroz.com.co/new/apr_public.php (consultado 9 oct. 2017).

FEDEARROZ (Federación Nacional de Arroceros). 2018. Importación de arroz a Colombia desde 2000 hasta 2019. FEDEARROZ, COL. http://www.fedearroz.com.co/new/importaciones.php (consultado 9 mayo 2019).

FINAGRO (Fondo para el Financiamiento del Sector Agropecuario). 2014. Sistema nacional de crédito agropecuario. Propuesta de reforma. Dirección de Desarrollo Rural Sostenible (DDRS), Bogotá, COL.

García, O. 2009. Carimagua: La investigación y el desarrollo en ecosistemas de baja fertilidad. Rev. Colomb. Cienc. Pecu. 22(1):74-78.

Gianessi, L.P., C.S. Silvers, S. Sankula, and J.E. Carpenter. 2002. Plant biotechnology: Current and potential impact for improving pest management in U.S. agriculture. An analysis of 40 case studies. The Heartland Institute, IL, USA. https:// www.heartland.org/_template-assets/documents/publications/15786.pdf (consultado 9 may. 2019).

Gómez, J. 2010. Manejo del suelo en la Altillanura de los Llanos Orientales de Colombia. En: V. Degiovanni et al., editores, Producción eco-eficiente del arroz en América Latina. CIAT, Cali, COL. p. 279-305.

Griliches, Z. 1957. Hybrid corn: An exploration in the economics of technological change. Econométrica 24:501-523. doi: $10.2307 / 1905380$

Guimarães, E.P., M. Châtel, Y. Ospina, y J. Borrero. 1995. Mejoramiento de arroz para suelos ácidos. Informe anual 1993A-1994B. CIAT, Cali, COL.

Hareau, G., B.F. Mills, y G.W. Norton. 2006. Arroz transgénico en Uruguay: un modelo de simulación para estimar los beneficios económicos potenciales. INIA, Montevideo, URY.

Moñux, D., B. Miranda, G. Aleixandre, y F.J. Gómez. 2005. Condicionantes políticos y problemas metodológicos en la evaluación de impacto social de las políticas de I+ D e innovación. Rev. Iberoam. Cienc. Tecnol. Soc. 2(4):173-200.

Perrin, R., R. Winkelman, y R. Anderson. 1983. Formulación de recomendaciones a partir de datos agronómicos: Un manual metodológico de evaluación económica. CIMMYT, México D.F., MEX.

Piraquive, G., M. Matamoros, E. Cespedes, y J. Rodríguez. 2018. Actualización de la tasa de rendimiento del capital en Colombia bajo la metodología de Harberger. Documento 487. Departamento Nacional de Planeación, COL. https:// colaboracion.dnp.gov.co/CDT/Estudios\%20Econmicos/487.pdf (consultado 8 mayo 2019).

Ramírez, M., H.J. Martínez, L.X. Ortíz, F.A. González, y C.A. Barrios. 2004. Respuestas de la oferta y la demanda agrícola en el marco de un TLC con Estados Unidos. IICA, Bogotá, COL.

Reyes-Hernández, M. 2002. Análisis económico de experimentos agrícolas con presupuestos parciales: re-enseñando el uso de este enfoque. La Calera 2(2):40-48.

Rivas, L., y C.J. Herrera. 2003. Impacto económico potencial en Colombia del uso de variedades transgénicas de yuca, resistentes al barrenador del tallo, Chilomina clarkei (Lepidoptera: Pyralidae). CIAT, Cali, COL. 
Rodríguez-Borray, G.A., R.A. Bautista, A.F. Dias-Avila, G. Stachetti-Rodrigues, y C. Espinosa. 2015. Diagnóstico socioeconómico y tecnológico de sistemas productivos agropecuarios de la altillanura colombiana: línea de base año 2011-2012. CORPOICA, Bogotá, COL.

Solé, R.S. 2011. Técnicas de evaluación de flujos de inversión: mitos y realidades. Cienc. Econ. 29:423-441.

Soler, D., y P. Hernández-Rodríguez. 2018. Desarrollos y perspectivas de investigación en la Orinoquía. Rev. Med. Vet. 36:7-13. doi:10.19052/mv.5167

Tapiero, A., S. Caicedo, J. Baquero, Y. Ospina, E. Guimarães, y M. Chatel. 2011. Arroz Corpoica Llanura 11 variedad mejorada de alta precocidad para los sistemas productivos de la altillanura plana. Plegable divulgativo $\mathrm{N}^{\circ} 60$. CORPOICA, Bogotá, COL.

Tirado, Y.C., y J.M. Barreto. 2014. Evaluación de la competitividad del arroz colombiano frente al estadounidense: Un análisis de la seguridad alimentaria en el marco del TLC. Tesis Lic., Universidad de Tolima, Ibagué, Tolima, COL.

World Bank. 2016. World development indicators. World bank group. http://data.worldbank.org/indicator/NY.GDS.TOTL.ZS (accessed Jun. 1, 2016). 